\title{
Cone effect in astronomical adaptive optics system investigated by a
}

\section{pure numerical simulation}

\author{
Hai-Xing Yan*, Han-Ling Wu, Shu-Shan Li and She Chen \\ (Institute of Mechanics, Chinese Academy of Sciences, Beijing 100080, China)
}

\begin{abstract}
It is well-known that cone effect or focus anisoplanatism is produced by the limited distance of a laser guide star (LGS) which is created within the Earth atmosphere and consequently located at a finite distance from the observer. In this paper, the cone effect of the LGS for different vertical profiles of the refractive index structure constant $C_{n}^{2}$ is numerically investigated by using a revised computer program of atmospheric propagation of optical wave and an adaptive optics (AO) system including dynamic control process. According to the practice, the overall tilt for the tilt-correction mirror is obtained from a natural star and the aberrated wavefront for phase correction of the deformable mirror is obtained from a LGS in our numerical simulation. It is surprisingly found that the effect of altitude of the LGS on the AO phase compensation effectiveness by using the commonly-available vertical profiles of $C_{n}^{2}$ and the lateral wind speed in the atmosphere is relatively weak, and the cone effect for some $C_{n}^{2}$ profiles is even negligible. It is found that the cone effect does not have obvious relationship with the turbulence strength, however, it depends on the vertical distribution profile of $C_{n}^{2}$ apparently. On the other hand, the cone effect depends on the vertical distribution of the lateral wind speed as well. In comparison to a longer wavelength, the cone effect becomes more obvious in the case of a shorter wavelength. In all cases concerned in this paper, an AO system by using a sodium guide star has almost same phase compensation effectiveness as that by using the astronomical target itself as a beacon. Effect of dynamic control process in an AO system on the cone effect is studied in this paper for the first time within our knowledge.
\end{abstract}

KEY WORDS: Adaptive optics system, Numerical simulation, Laser guide star, Cone effect, Adaptive optics telescope, Phase compensation effectiveness, vertical profile of refractive index structure constant

\section{INTRODUCTION}

An adaptive optics (AO) telescope can greatly improve the astronomical observation on the Earth. However, knowledge of the aberrated wavefront is the necessary input for an AO system and the AO system needs a bright enough beacon (in astronomy it is called as a guide star) within the isoplanatic field for the real-time measurement and correction of the wavefront. ${ }^{1,2}$ This requirement limits the use of AO for astronomy because in most cases it is quite difficult to find a bright enough guide star within the isoplanatic field of the interested target. This was why Foy and Labeyrie suggested in 1985 to use a laser to generate artificial guide star at arbitrary positions on the sky: ${ }^{3}$ the laser is focused into the atmosphere at small angle from the target object, and backscattering light, i.e. the "laser guide star (LGS)", is used by the wavefront sensor to measure the aberrated wavefront. Either Rayleigh or Mie scattering off air molecules at moderate altitudes (up to about $20 \mathrm{~km}$ ) and resonant scattering by sodium (Na) atoms at an altitude of

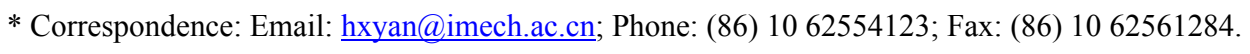


about $90 \mathrm{~km}$ are powerful ways to obtain the $\mathrm{LGS}^{4}$

One of two primary problems introduced by the LGS is called as cone effect. The cone effect comes from the finite height of the artificial guide star above the telescope. The light rays from the astronomical target, which is at infinite or very great distance, form a parallel bundle and pass through the atmosphere as a cylinder, while the LGS emits a spherical wavefront and the rays from the LGS trace out a cone. The turbulence above the LGS is not sampled at all, and the difference in the propagation paths (i.e. between the cylinder and the cone) leads to an incorrect sampling of the turbulence below the LGS. For the sodium guide stars, which are formed well above the troposphere, the first of these two effects is negligible, and it is thought that the second effect is less severe than for Rayleigh guide stars. The cone effect induces a systemic erroneous correction and thus limits improvement of the image quality. The cone effect becomes more critical toward shorter wavelength, and for larger telescope apertures.

During the recent 30 years, the cone effect for the LGS and solutions for eliminating it in the adaptive optics astronomy have been widely investigated. ${ }^{2,4,5}$ However, almost all of these investigations are by means of the analytical theory. For example, in China, Shen investigated an important problem - modal decomposition of the anisoplanatic errors in his doctoral thesis. ${ }^{6}$ Based on the Zernike polynomials, the statistical correlation of random wavefronts between the LGS and the target was studied, and the focus anisoplanatism and the angular anisoplanatism were unified. The mathematical expressions for modal decomposition and the analytic relationships for modal anisoplanatism of planar and spherical wave were presented. Viard and his colleagues studied the four-LGS method for improving the image quality when an AO system is used in an astronomical telescope. ${ }^{7}$ Based on an analytical study, the residual cone effect with four LGSs was investigated and presented. Furthermore, in order to overcome the cone effect Buscher el al. proposed an alternative method to sense the wavefront distortion produced during the beam's return passage of a LGS. ${ }^{8}$ A key advantage of their method is that a parallel beam is used, and therefore the wavefront measurements will not suffer from the cone effect.

On the other hand, generally, an AO system can be divided into three parts: wavefront detection, wavefront reconstruction and wavefront correction. Moreover, there are three important factors to affect the performances of the $\mathrm{AO}$ system. The first of them is the limited spatial bandwidth resulted from the limited numbers of sub-apertures in the wavefront sensor for wavefront detection and actuators in the deformable mirror for wavefront correction. The second is effects of noise and detection error. The third is the limited temporal bandwidth resulted from the limited response speed for wavefront detection, reconstruction and correction. The present authors have been pursuing modeling and numerical simulation of atmospheric propagation of optical waves and an AO system since 1988. ${ }^{9-14}$ In Ref. [9], a comprehensive theoretical model has been developed and coded into a numerical algorithm. A numerical simulation of the atmospheric propagation of an optical wave in the turbulence and a numerical simulation of the operation of an AO system in a static state were combined. The distorted wavefront after propagating through the atmospheric turbulence is detected, reconstructed and corrected by the $\mathrm{AO}$ system. In that paper, the direct wavefront gradient control method was used to reconstruct the wavefront phase and the long-exposure Strehl ratio was used to evaluate the performances of the AO system. In Ref. [10], a theoretical model of numerical simulation of an AO system by modal wavefront reconstruction (i.e. by means of the Zernike polynomials expansion) was presented and a corresponding computer program was compiled. A numerical simulation investigation of an $\mathrm{AO}$ system by means of the modal wavefront reconstruction was carried out in that paper. The first factor to affect the AO system performances, i.e. the effect of limited spatial bandwidth was investigated in Refs. [9,10]. Refs. [11,12] focused on the effects of noise and detection error and on the dynamic control process and frequency response characteristics in an AO system, respectively. Thus, a comprehensive modeling 
and numerical simulation of an AO system were completed. Based on our theoretical model and computer program, the effects of reconstruction matrix and controller coefficients on performances of an $\mathrm{AO}$ system and the interaction of the effects of noise and detection error with the dynamic control process in an AO system were investigated by a pure numerical simulation. ${ }^{13,14}$

In our preliminary paper, ${ }^{15}$ the cone effect was investigated by means of a pure numerical simulation of atmospheric propagation of optical wave and an adaptive optics (AO) system including dynamic control process. It should be noted that in all the previous investigations of the cone effect, analytical formulae have been utilized and the dynamic control process in an AO system has not been included. However, in practice an AO system must include the dynamic control process as a necessary portion of the AO system and the dynamic control process might have important influence on the cone effect, i.e. the cone effect for an AO system including the dynamic control process might have some important differences in comparison to that without the dynamic control process. In light of these, our preliminary numerical simulation investigation of the cone effect was carried out. However, there were some defects in that paper. One of the important defects is that a LGS is used to obtain the phase knowledge both for the tilt-correction mirror and for the deformable mirror, i.e. both the overall tilt and the higher order aberrated wavefront. In practice, it is impossible to obtain the overall tilt of the aberrated wavefront from detecting a LGS.

By contrast, in this paper, various conditions in the practical application of wave propagation through the turbulent atmosphere and an AO system with a PI (proportional plus integral) controller are included in an analogous way. That is, the knowledge of overall tilt of the aberrated wavefront for phase correction of the tilt-correction mirror is detected from either the astronomical target itself or another astronomical target within the isoplanatic field of the interested target, and the knowledge of higher order aberrated wavefront for phase correction of the deformable mirror is detected from a LGS. Thus, it is possible to investigate effects of the angular anisoplanatism of the overall tilt detection on an astronomical AO system. Furthermore, a complete numerical simulation for an astronomical imaging system is realized in this paper. In comparison to the preliminary paper, more computational results under different computational conditions are presented in this paper.

\section{NEMERICAL SIMULATION}

The propagation of an optical wave through the turbulent atmosphere is a key problem in this investigation. As in Ref. [9], the multiple phase screen (PS) model is used to describe the turbulent media. The main idea is as follows. The effect of turbulence on an optical wave is expressed in terms of random change in the refractive index. The propagation path through the turbulent media can be divided into several segments which may have different lengths. It is thought that each segment may deform the phase of the optical wave independently. The contribution of the turbulent media segment to the phase of the optical wave can be "pressed" into a very thin phase screen and added to the initial phase of the wave. It is assumed that the phase screen does not have a significant influence on the amplitude of the wave. The amplitude of the optical wave is changed only in the propagation process of the wave with the deformed phase. It means that changes in phase and amplitude of an optical wave can be treated separately. At the position of the next phase screen, the wave with changed amplitude is modified by the new phase screen and so on. This process continues until the wave reaches the target.

Following Dr. Li of the Institute of Optics and Electronics, Chinese Academy of Sciences, ${ }^{16}$ five atmospheric models, i.e. distribution profiles of $C_{n}^{2}$ vs. altitude, are used in this paper. The well-known Hufnagel-Valley (HV) models are included. Equations relating the altitude $h$ (in meter) to $C_{n}^{2}$ are: 
HV0: $C_{n}^{2}=8.16 \times 10^{-54} h^{10} e^{-h / 1000}+3.02 \times 10^{-17} e^{-h / 1500}+1.43 \times 10^{-14} e^{-h / 100}$

Modified HV1: $\quad C_{n}^{2}=8.16 \times 10^{-54} h^{10} e^{-h / 1000}+3.02 \times 10^{-17} e^{-h / 1500}+1.9 \times 10^{-15} e^{-h / 100}$

HV-21: $C_{n}^{2}=5.94 \times 10^{-53}(\omega / 27)^{2} h^{10} e^{-h / 1000}+2.7 \times 10^{-16} e^{-h / 1500}+A e^{-h / 100}$

$$
\omega=21 \mathrm{~m} / \mathrm{s}, A=1.7 \times 10^{-14}
$$

HFD $C_{n}^{2}=5.3 \times 10^{-50} h^{10} e^{-h / 610}+4 \times 10^{-15} e^{-h / 300}+1.8 \times 10^{-16} e^{-h / 430}$

SLC-day: $\quad C_{n}^{2}=\left\{\begin{array}{cc}0 & 0 m<h<19 m \\ 4.008 \times 10^{-13} h^{-1.054} & 19 m<h<230 m \\ 1.3 \times 10^{-15} & 230 m<h<850 m \\ 6.352 \times 10^{-7} h^{-2.966} & 850 m<h<7000 m \\ 6.209 \times 10^{-16} h^{-0.6229} & 7000 m<h<30000 m\end{array}\right.$

A common feature of all these models is that $C_{n}^{2}$ is largest on the ground; as altitude increases, $C_{n}^{2}$ decreases rapidly; there is a region having a weak turbulence around the altitude of $2 \mathrm{~km}$ for most of the models; and there is a region having a stronger turbulence around the altitude of $5-10 \mathrm{~km}$. According to this feature, we select an arrangement of the multiple phase screens. After numerical experiment, 17 phase screens are chosen, and their distribution is as follows: 5 phase screens in 0-500 m, divided equally, i.e. one per $100 \mathrm{~m} ; 3$ phase screens in $500-2000 \mathrm{~m}$, divided equally, i.e. one per $500 \mathrm{~m} ; 5$ phase screens in 2-12 km, divided equally, i.e. one per $2 \mathrm{~km}$; 4 phase screens in 12-30 km, divided equally, i.e. one per $4.5 \mathrm{~km}$; and the atmospheric turbulence is omitted in the region above $30 \mathrm{~km}$. This arrangement is based on the following consideration: fully utilizing not too many phase screens to obtain more accurate computational results in view of that the computer hours are proportional to the number of phase screens; utilizing more phase screens in the region having stronger turbulence and rapid variation of $C_{n}^{2}$ vs. altitude; utilizing less and less phase screens as the turbulence getting weaker and the variation of $C_{n}^{2}$ vs. altitude getting slower.

The average $C_{n}^{2}$ at positions of the 17 phase screens and the atmospheric coherence length $r_{0-I R}$ of the whole propagation path for a near infrared (IR) wavelength $(1.5 \mu \mathrm{m})$ by using these five models are summarized in Table 1 . The corresponding figure of the $C_{n}^{2}$ distributions vs. altitude for the five atmospheric models is shown in Fig. 1.

It is shown from Table 1 and Fig. 1 that the $C_{n}^{2}$ magnitudes and distributions for the five models are quite different. For $r_{0-I R}$ of the whole propagation path, value for model HV1 is larger, i.e. the atmospheric turbulence for it is weaker. In fact, the distribution of $C_{n}^{2}$ vs. altitude has more important influence on the astronomical observation on the ground which influence is the main interested topic in this paper.

For the distribution of lateral wind speed vs. altitude, a commonly used model of the wind speed is used in this paper. The equation relating the lateral wind speed to the altitude $v_{w}(h)$ is expressed as:

$$
v_{w}(h)=v_{g}+30 \exp \left\{-[(h-9400) / 4800]^{2}\right\} \quad(\mathrm{m} / \mathrm{s}),
$$

where $v_{g}=8 \mathrm{~m} / \mathrm{s}$ for HV-21 model and $v_{g}=5 \mathrm{~m} / \mathrm{s}$ for others. The distribution of the wind speed (when $v_{g}=5 \mathrm{~m} / \mathrm{s}$ ) vs. 
the altitude is shown in Table 1 as well. Its corresponding figure is Fig. 2.

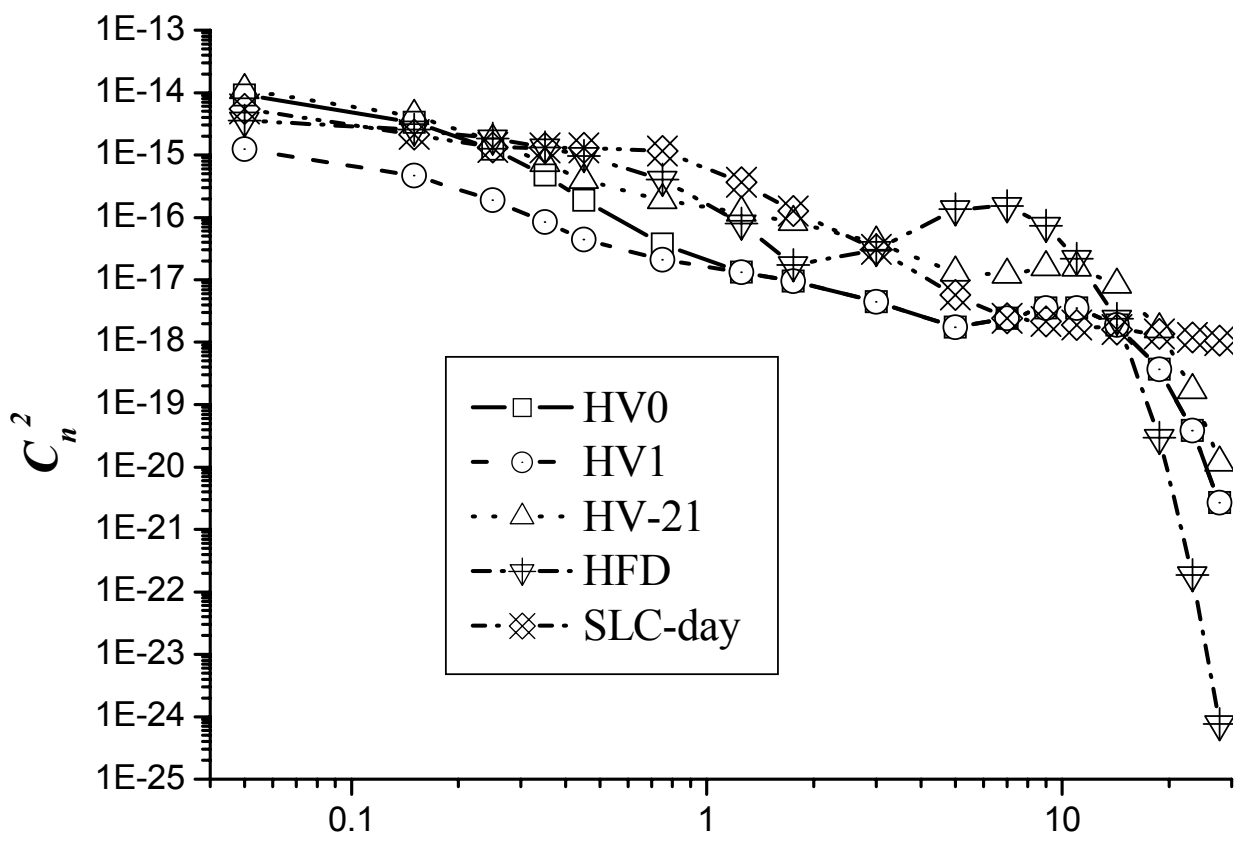

Altitude (km)

\section{Fig. 1 Distributions of $C_{n}^{2}$ vs. altitude $\boldsymbol{h}$ for five atmospheric turbulence models.}

In another aspect, an exemplary AO system is utilized in this paper. The configuration of the deformable mirror (DM) actuators and the Hartmann-Shack (HS) sensor subapetures in the AO system is same as that in Ref. [12]. This AO system includes a HS wavefront sensor with 48 subapertures and the number of actuator in the deformable mirror is 61 . The diameter of the telescope is assumed to be 0.6 meter. The coupling factor between adjacent actuators is taken as 0.15 . In present paper, the dynamic control process of an $\mathrm{AO}$ system is included, but the effects of noise and detection error are not included.

Some other concrete simulation conditions are as

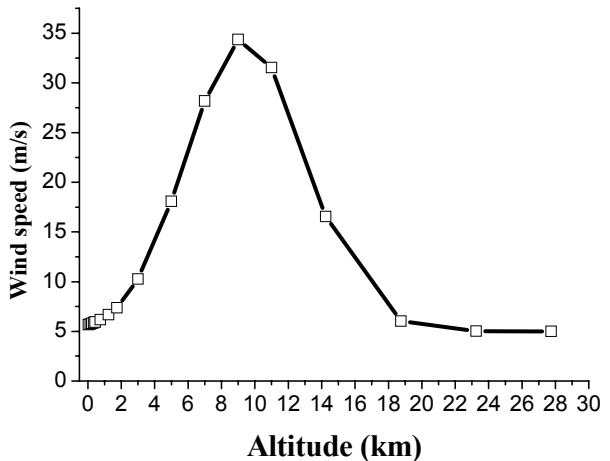

Fig. 2 Distributions of the wind speed $v_{w}$ vs. altitude $h$.

follows. An optical wave propagation scene of a geostationary satellite which altitude is about $36 \times 10^{3} \mathrm{~km}$ is chosen in this paper. The altitudes of the Rayleigh guide star are chosen as 5,10, and $20 \mathrm{~km}$; and the altitude of the sodium guide star is chosen as $90 \mathrm{~km}$. The beacon wavelength of the Rayleigh guide star is taken as $532 \mathrm{~nm}$; and the beacon wavelength of the sodium guide star is taken as $589 \mathrm{~nm}$ as in the practice. The knowledge of higher order aberrated wavefront for phase correction of the deformable mirror is detected from a LGS. On the other hand, the knowledge of 
overall tilt of the aberrated wavefront for phase correction of the tilt-correction mirror is detected from either the geostationary satellite itself or another astronomical target within the isoplanatic field of the geostationary satellite. The light wavelength for detecting the overall tilt is taken as $0.55 \mu \mathrm{m}$.

Table 1 Average $C_{n}^{2}$ and average $v_{w}$ at positions of 17 phase screens and the atmospheric coherence length $r_{0-I R}$ of the whole propagation path for $1.5 \mu \mathrm{m}$ by using five atmospheric models

\begin{tabular}{|c|c|c|c|c|c|c|c|}
\hline \multicolumn{2}{|c|}{ Model } & HV0 & HV1 & HV-21 & HFD & SLC-day \\
\hline$r_{0-I R}(\mathrm{~cm})$ & 23.508 & 66.659 & 18.540 & 19.169 & 19.314 \\
\hline $\begin{array}{c}h \\
(\mathrm{~km})\end{array}$ & $\begin{array}{c}v_{w}(h) \\
(\mathrm{m} / \mathrm{s})\end{array}$ & \multicolumn{5}{|c|}{ Average $C_{n}^{2}\left(\mathrm{~m}^{-2 / 3}\right)$} \\
\hline 0.05 & 5.675 & $0.9072 \mathrm{E}-14$ & $0.1231 \mathrm{E}-14$ & $0.1101 \mathrm{E}-13$ & $0.3562 \mathrm{E}-14$ & $0.5456 \mathrm{E}-14$ \\
\hline 0.15 & 5.732 & $0.3354 \mathrm{E}-14$ & $0.4693 \mathrm{E}-15$ & $0.4199 \mathrm{E}-14$ & $0.2565 \mathrm{E}-14$ & $0.2127 \mathrm{E}-14$ \\
\hline 0.25 & 5.793 & $0.1249 \mathrm{E}-14$ & $0.1882 \mathrm{E}-15$ & $0.1684 \mathrm{E}-14$ & $0.1847 \mathrm{E}-14$ & $0.1330 \mathrm{E}-14$ \\
\hline 0.35 & 5.858 & $0.4741 \mathrm{E}-15$ & $0.8374 \mathrm{E}-16$ & $0.7491 \mathrm{E}-15$ & $0.1331 \mathrm{E}-14$ & $0.1300 \mathrm{E}-14$ \\
\hline 0.45 & 5.928 & $0.1880 \mathrm{E}-15$ & $0.4438 \mathrm{E}-16$ & $0.3970 \mathrm{E}-15$ & $0.9601 \mathrm{E}-15$ & $0.1300 \mathrm{E}-14$ \\
\hline 0.75 & 6.172 & $0.3760 \mathrm{E}-16$ & $0.2095 \mathrm{E}-16$ & $0.1874 \mathrm{E}-15$ & $0.4011 \mathrm{E}-15$ & $0.1160 \mathrm{E}-14$ \\
\hline 1.25 & 6.686 & $0.1332 \mathrm{E}-16$ & $0.1320 \mathrm{E}-16$ & $0.1180 \mathrm{E}-15$ & $0.7997 \mathrm{E}-16$ & $0.3632 \mathrm{E}-15$ \\
\hline 1.75 & 7.375 & $0.9449 \mathrm{E}-17$ & $0.9449 \mathrm{E}-17$ & $0.8447 \mathrm{E}-16$ & $0.1730 \mathrm{E}-16$ & $0.1273 \mathrm{E}-15$ \\
\hline 3.00 & 10.26 & $0.4439 \mathrm{E}-17$ & $0.4439 \mathrm{E}-17$ & $0.3950 \mathrm{E}-16$ & $0.3021 \mathrm{E}-16$ & $0.3073 \mathrm{E}-16$ \\
\hline 5.00 & 18.07 & $0.1748 \mathrm{E}-17$ & $0.1748 \mathrm{E}-17$ & $0.1296 \mathrm{E}-16$ & $0.1366 \mathrm{E}-15$ & $0.5704 \mathrm{E}-17$ \\
\hline 7.00 & 28.19 & $0.2400 \mathrm{E}-17$ & $0.2400 \mathrm{E}-17$ & $0.1196 \mathrm{E}-16$ & $0.1513 \mathrm{E}-15$ & $0.2411 \mathrm{E}-17$ \\
\hline 9.00 & 34.37 & $0.3528 \mathrm{E}-17$ & $0.3528 \mathrm{E}-17$ & $0.1590 \mathrm{E}-16$ & $0.7408 \mathrm{E}-16$ & $0.2142 \mathrm{E}-17$ \\
\hline 11.00 & 31.55 & $0.3512 \mathrm{E}-17$ & $0.3512 \mathrm{E}-17$ & $0.1556 \mathrm{E}-16$ & $0.2178 \mathrm{E}-16$ & $0.1889 \mathrm{E}-17$ \\
\hline 14.25 & 16.55 & $0.1881 \mathrm{E}-17$ & $0.1881 \mathrm{E}-17$ & $0.8298 \mathrm{E}-17$ & $0.2367 \mathrm{E}-17$ & $0.1612 \mathrm{E}-17$ \\
\hline 18.75 & 6.016 & $0.3662 \mathrm{E}-18$ & $0.3662 \mathrm{E}-18$ & $0.1613 \mathrm{E}-17$ & $0.2946 \mathrm{E}-19$ & $0.1357 \mathrm{E}-17$ \\
\hline 23.25 & 5.018 & $0.3833 \mathrm{E}-19$ & $0.3833 \mathrm{E}-19$ & $0.1688 \mathrm{E}-18$ & $0.1856 \mathrm{E}-21$ & $0.1185 \mathrm{E}-17$ \\
\hline 27.75 & 5.000 & $0.2677 \mathrm{E}-20$ & $0.2676 \mathrm{E}-20$ & $0.1177 \mathrm{E}-19$ & $0.7597 \mathrm{E}-24$ & $0.1061 \mathrm{E}-17$ \\
\hline
\end{tabular}

In a practical AO system, a controller for controlling the dynamic process must be included and a dynamic iteration computation of thousands time steps must be carried out. ${ }^{12,13}$ A difference equation

$$
U_{n}=a_{1} U_{n-1}+a_{2} U_{n-2}+a_{3} U_{n-3}+\Lambda+b_{1} E_{n}+b_{2} E_{n-1}+b_{3} E_{n-2}+\Lambda
$$

connects the corrected wavefront at the $n$th time step $U_{n}$ with the corrected wavefronts at the foregoing time steps $U_{n-1}$, $U_{n-2}, \ldots$ and the residual wavefronts at the present time step and the foregoing time steps $E_{n}, E_{n-1}, E_{n-2}, \ldots$ A different controller shows different performances in terms of the difference of this connection, i.e. in terms of the different coefficients in this equation. A simpler PI (proportional plus integral) controller is utilized and the controller coefficients $a_{l}=0.94, b_{1}=0.50$, others $=0$ are chosen in this paper. 3000 dynamic iterations are utilized to obtain the statistically average simulation results in this paper.

\section{RESULT AND DISCUSSION}

We propose to use a parameter $S R$ as an evaluation parameter to express the phase compensation effectiveness of an $\mathrm{AO}$ system. The $S R$ is defined as: 


$$
S R=S T R C C \text { (closed loop) / STRCC (open loop) }
$$

wherein STRCC (closed loop) is the Strehl ratio STRCC for the AO system with a closed loop (i.e. having the phase compensation of the AO system), and STRCC (open loop) is the STRCC for the AO system with an open loop (i.e. without the phase compensation). The $S T R C C$ is defined as a ratio of the optical energy within a circle around the centroid (optical center) with a radius of the first dark ring in the Airy pattern after propagations through the turbulent medium to that through a vacuum. ${ }^{9}$ The long-exposure STRCC is utilized in this paper. In the numerical simulation of an AO system including dynamic control process, a large number of patterns at target corresponding to different time steps of dynamic iteration are accumulated to obtain a long-exposure pattern. In calculating the long-exposure STRCC, the centroid and the Strehl ratio are determined from the accumulated long-exposure pattern at target.

\section{(1) Effect of different angular anisoplanatisms for the overall tilt detection}

Choosing the zenith angle of 0 degree and light wavelength of $1.5 \mu \mathrm{m}$, and by using 61 units $\mathrm{AO}$ system having sampling frequency of $1000 \mathrm{~Hz}$, computational results of the numerical simulation for five atmospheric turbulence models in the case of angular anisoplanatism of $0 \mu \mathrm{r}$ for the overall tilt detection are summarized in Table 2. As mentioned above, the dynamic control process of an AO system is included in the simulation, and the $S R$ is chosen as the evaluation parameter of the phase compensation effectiveness of an AO system. Its corresponding figure is Fig. 3.

Table 2 Dynamic iteration results $S R$ vs. distance of the beacon in the case of angular anisoplanatism of $0 \mu r$ for the overall tilt detection, a wavelength of $1.5 \mu \mathrm{m}$ and zenith angle of 0 degree by using 61 units $\mathrm{AO}$ system having $1000 \mathrm{~Hz}$ sampling frequency

\begin{tabular}{|c|c|c|c|c|c|c|}
\hline $\begin{array}{c}\text { Distance } \\
(\mathrm{km})\end{array}$ & $\begin{array}{c}\text { Number } \\
\text { of PS }\end{array}$ & HV0 & HV1 & HV-21 & HFD & SLC-day \\
\hline \multicolumn{2}{|c|}{$r_{0-I R}(\mathrm{~cm})$} & 23.508 & 66.659 & 18.540 & 19.169 & 19.314 \\
\hline 5 & 9 & 1.934 & 1.113 & 2.351 & 1.803 & 2.308 \\
\hline 10 & 12 & 1.943 & 1.117 & 2.392 & 1.878 & 2.423 \\
\hline 20 & 15 & 1.947 & 1.119 & 2.410 & 1.926 & 2.464 \\
\hline 90 & 17 & 1.949 & 1.121 & 2.413 & 1.936 & 2.485 \\
\hline 36000 & 17 & 1.949 & 1.125 & 2.422 & 1.984 & 2.502 \\
\hline
\end{tabular}

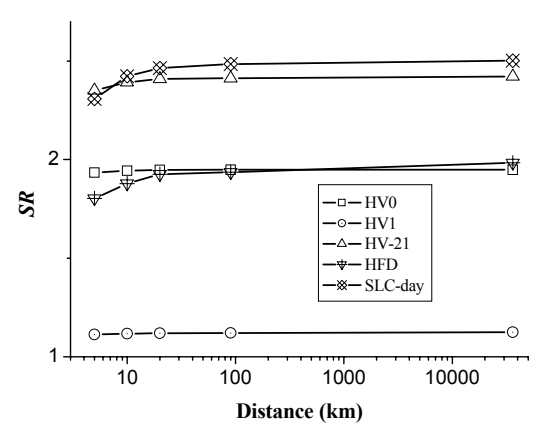

Fig. $3 S R$ vs. beacon distance for angular anisoplanatism of $0 \mu r$

It is surprisingly found from Table 2 and Fig. 3 that the effect of altitudes of the beacon (LGS or the astronomical target itself) on the $\mathrm{AO}$ phase compensation effectiveness by using the commonly-available vertical profiles of the refractive index structure constant $C_{n}^{2}$ in the atmosphere is relatively weak, and the cone effect for some profiles is even negligible. For example, the cone effect for model HV0 and HV1 is almost negligible, i.e. the curves of $S R$ vs. beacon altitude for these models are almost flat. (See Fig. 3)

It is found that the cone effect does not have obvious relationship with the turbulence strength. The atmospheric coherence lengths $r_{0}$ for models HV0 and HV1 are quite different (see Table 1 or 2), but the cone effect for these models is similarly negligible, although $S R$ (the phase compensation effectiveness) has obvious difference for them. However, the cone effect depends on the distribution profiles of $C_{n}^{2}$ obviously. When $C_{n}^{2}$ distribution of an atmospheric turbulence model is that $C_{n}^{2}$ is relatively large at lower altitude, especially $C_{n}^{2}$ is very large near the ground, and $C_{n}^{2}$ decreases rapidly as altitude increases, the cone effect for this model is weaker. This is the case for models HV0 and 
HV1. On the contrary, if $C_{n}^{2}$ is relatively small at lower altitude, and especially $C_{n}^{2}$ decreases slowly as altitude increases, the cone effect for this model is more obvious. For example, this is the case of model SLC-day. The reason for these can be understood as follows. As mentioned above, there are two sources to produce the cone effect: the first is that the turbulence above the LGS is not sampled at all; the second is the difference in propagation paths of the astronomical target and the beacon (i.e. the difference between a cylinder and a cone). Because the atmospheric turbulence in the region of higher altitudes is relatively weak, it can be imagined that the influence of the first source has less importance. As far as the second source is concerned, the difference between a cylinder and a cone is smaller at lower altitudes and this difference gets larger and larger as the altitude increases. Thus, in the case that the atmospheric turbulence at higher altitudes is not very weak, the cone effect should be more obvious.

Table 3 Dynamic iteration results $S R$ vs. distance of the beacon in the case of angular anisoplanatism of $300 \mu r$ for the overall tilt detection, other conditions are same as Table 2

\begin{tabular}{|c|c|c|c|c|c|c|}
\hline $\begin{array}{c}\text { Distance } \\
(\mathrm{km})\end{array}$ & $\begin{array}{c}\text { Number } \\
\text { of PS }\end{array}$ & HV0 & HV1 & HV-21 & HFD & SLC-day \\
\hline 5 & 9 & 1.896 & 1.101 & 1.931 & 1.327 & 1.985 \\
\hline 10 & 12 & 1.905 & 1.105 & 1.970 & 1.374 & 2.087 \\
\hline 20 & 15 & 1.910 & 1.108 & 1.988 & 1.411 & 2.147 \\
\hline 90 & 17 & 1.912 & 1.110 & 1.998 & 1.409 & 2.153 \\
\hline 36000 & 17 & 1.911 & 1.115 & 2.012 & 1.442 & 2.164 \\
\hline
\end{tabular}

Table 4 Dynamic iteration results $S R$ vs. distance of the beacon in the case of angular anisoplanatism of $600 \mu \mathrm{r}$ for the overall tilt detection, other conditions are same as Table 2

\begin{tabular}{|c|c|c|c|c|c|c|}
\hline $\begin{array}{c}\text { Distance } \\
(\mathrm{km})\end{array}$ & $\begin{array}{c}\text { Number } \\
\text { of PS }\end{array}$ & HV0 & HV1 & HV-21 & HFD & SLC-day \\
\hline 5 & 9 & 1.882 & 1.096 & 1.914 & 1.214 & 1.691 \\
\hline 10 & 12 & 1.891 & 1.102 & 1.948 & 1.254 & 1.773 \\
\hline 20 & 15 & 1.896 & 1.104 & 1.965 & 1.280 & 1.824 \\
\hline 90 & 17 & 1.898 & 1.108 & 1.986 & 1.291 & 1.827 \\
\hline 36000 & 17 & 1.897 & 1.113 & 1.995 & 1.315 & 1.828 \\
\hline
\end{tabular}

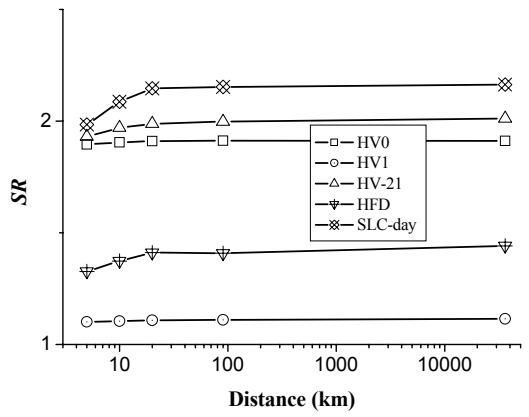

Fig. $4 S R$ vs. beacon distance for angular anisoplanatism of $\mathbf{3 0 0} \mu \mathrm{r}$

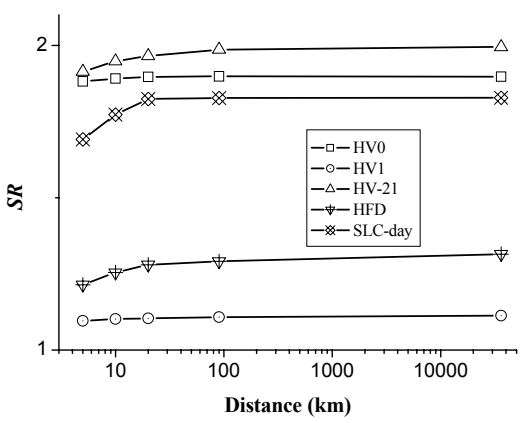

Fig. $5 S R$ vs. beacon distance for angular anisoplanatism of $600 \mu r$

On the other hand, the cone effect depends on the vertical distribution of the lateral wind speed as well. As mentioned above, the numerical simulation results presented in this paper include the effect of dynamic control process in an AO system. For a static target such as a geostationary satellite, the effect of dynamic control process mainly comes from the lateral wind in the atmosphere. It can be seen from Table 1 and Fig. 2 that the lateral wind speed in the atmosphere has a peak around $10 \mathrm{~km}$ altitude. Thus, in the case that the atmospheric turbulence at higher altitudes is not very weak, the cone effect could be more obvious as well.

Dynamic iteration results $S R$ in the case of angular anisoplanatism of $300 \mu r$ and $600 \mu r$ for the overall tilt detection are summarized in Tables 3 and 4. Their corresponding figures are Figs. 4 and 5. It is shown that the cone effect does not 
have obvious change as the angular anisoplanatism for the overall tilt detection gets larger although the phase compensation effectiveness $S R$ gets smaller.

\section{(2) In the case of different zenith angles}

Choosing the zenith angles of 30, 45 and 60 degree, the Dynamic iteration results $S R$ are summarized in Tables 5-7, respectively. Their corresponding figures are Figs. 6-8. It can be seen that as the zenith angle increases, $r_{0-I R}$ becomes

Table $5 S R$ vs. distance of the beacon in the case of zenith angle of 30 degree, other conditions are same as Table 3

\begin{tabular}{|c|c|c|c|c|c|c|}
\hline $\begin{array}{c}\text { Distance } \\
(\mathrm{km})\end{array}$ & $\begin{array}{c}\text { Number } \\
\text { of PS }\end{array}$ & HV0 & HV1 & HV-21 & HFD & SLC-day \\
\hline \multicolumn{2}{|c|}{$R_{0-I R}(\mathrm{~cm})$} & 21.564 & 61.147 & 17.007 & 17.584 & 17.717 \\
\hline 5 & 9 & 2.017 & 1.124 & 2.021 & 1.291 & 2.028 \\
\hline 10 & 11 & 2.029 & 1.129 & 2.063 & 1.333 & 2.161 \\
\hline 20 & 14 & 2.036 & 1.132 & 2.093 & 1.376 & 2.210 \\
\hline 103.68 & 17 & 2.040 & 1.135 & 2.111 & 1.398 & 2.244 \\
\hline 36734 & 17 & 2.041 & 1.141 & 2.119 & 1.458 & 2.260 \\
\hline
\end{tabular}

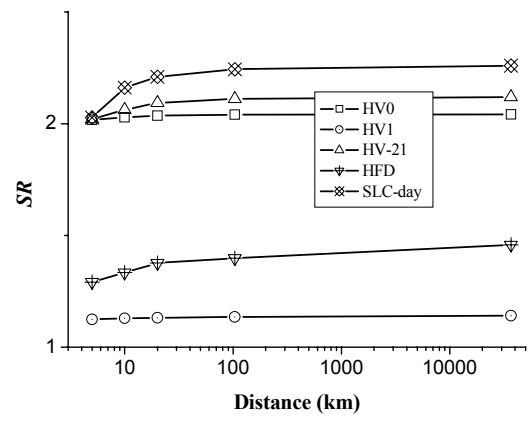

Fig. $6 S R$ vs. beacon distance for zenith angle of 30 degree

Table $6 S R$ vs. distance of the beacon in the case of zenith angle of 45 degree, other conditions are same as Table 3

\begin{tabular}{|c|c|c|c|c|c|c|}
\hline $\begin{array}{c}\text { Distance } \\
(\mathrm{km})\end{array}$ & $\begin{array}{c}\text { Number } \\
\text { of PS }\end{array}$ & HV0 & HV1 & HV-21 & HFD & SLC-day \\
\hline \multicolumn{2}{|c|}{$R_{0-I R}(\mathrm{~cm})$} & 19.094 & 54.144 & 15.059 & 15.570 & 15.688 \\
\hline 5 & 9 & 2.223 & 1.149 & 2.177 & 1.291 & 1.950 \\
\hline 10 & 11 & 2.243 & 1.155 & 2.234 & 1.339 & 2.139 \\
\hline 20 & 13 & 2.255 & 1.160 & 2.276 & 1.392 & 2.270 \\
\hline 126.41 & 17 & 2.261 & 1.165 & 2.296 & 1.431 & 2.273 \\
\hline 37626 & 17 & 2.262 & 1.172 & 2.326 & 1.471 & 2.292 \\
\hline
\end{tabular}

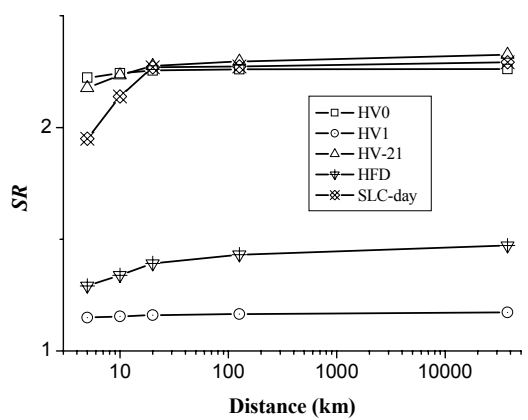

Fig. $7 S R$ vs. beacon distance for zenith

Table 7 Dynamic iteration results $S R$ vs. distance of the beacon in the case of zenith angle of 60 degree, other conditions are same as

Table 3

\begin{tabular}{|c|c|c|c|c|c|c|}
\hline $\begin{array}{c}\text { Distance } \\
(\mathrm{km})\end{array}$ & $\begin{array}{c}\text { Number } \\
\text { of PS }\end{array}$ & HV0 & HV1 & HV-21 & HFD & SLC-day \\
\hline \multicolumn{2}{|c|}{$R_{0-I R}(\mathrm{~cm})$} & 15.51 & 43.98 & 12.23 & 12.65 & 12.74 \\
\hline 5 & 8 & 2.783 & 1.231 & 2.367 & 1.529 & 2.325 \\
\hline 10 & 9 & 2.829 & 1.240 & 2.444 & 1.604 & 2.677 \\
\hline 20 & 12 & 2.853 & 1.247 & 2.511 & 1.666 & 3.004 \\
\hline 176.40 & 17 & 2.879 & 1.260 & 2.556 & 1.738 & 3.004 \\
\hline 38825 & 17 & 2.881 & 1.266 & 2.617 & 1.757 & 3.033 \\
\hline
\end{tabular}

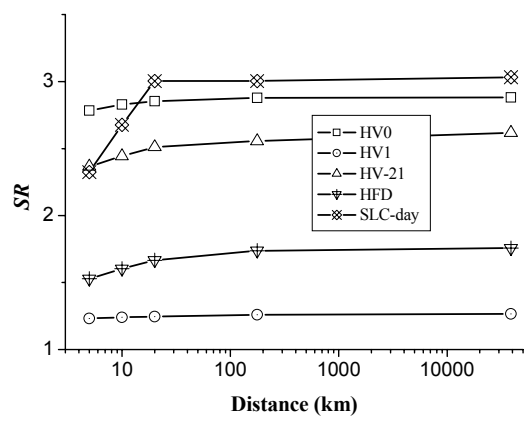

Fig. 8 SR vs. beacon distance for zenith angle of 60 degree

smaller, i.e. the atmospheric turbulence strength becomes stronger, and phase compensation effectiveness $S R$ becomes 
larger. Further, it can be seen from Tables 5-7 and Figs. 6-8 that the cone effect becomes more obvious as the zenith angle increases.

\section{(3) In the case of different $\mathrm{AO}$ system sampling frequency}

When the sampling frequency in the AO system increases, $S R$ results are summarized in Tables 8 and 9. Their corresponding figures are Figs. 9 and 10. It can be seen that as the AO sampling frequency increases, the phase compensation effectiveness $S R$ becomes larger, but change in the cone effect is not very obvious.

Table 8 Dynamic iteration results $S R$ vs. distance of the beacon in the case of $\mathrm{AO}$ system sampling frequency of $2000 \mathrm{~Hz}$, other conditions are same as Table 3

\begin{tabular}{|c|c|c|c|c|c|c|}
\hline $\begin{array}{c}\text { Distance } \\
(\mathrm{km})\end{array}$ & $\begin{array}{c}\text { Number } \\
\text { of PS }\end{array}$ & HV0 & HV1 & HV-21 & HFD & SLC-day \\
\hline 5 & 9 & 1.960 & 1.116 & 2.107 & 1.352 & 1.926 \\
\hline 10 & 12 & 1.970 & 1.120 & 2.158 & 1.387 & 2.028 \\
\hline 20 & 15 & 1.977 & 1.124 & 2.198 & 1.475 & 2.086 \\
\hline 90 & 17 & 1.986 & 1.129 & 2.237 & 1.548 & 2.102 \\
\hline 36000 & 17 & 1.990 & 1.131 & 2.253 & 1.589 & 2.118 \\
\hline
\end{tabular}

Table 9 Dynamic iteration results $S R$ vs. distance of the beacon in the case of $\mathrm{AO}$ system sampling frequency of $4000 \mathrm{~Hz}$, other conditions are same as Table 3

\begin{tabular}{|c|c|c|c|c|c|c|}
\hline $\begin{array}{c}\text { Distance } \\
(\mathrm{km})\end{array}$ & $\begin{array}{c}\text { Number } \\
\text { of PS }\end{array}$ & HV0 & HV1 & HV-21 & HFD & SLC-day \\
\hline 5 & 9 & 1.976 & 1.120 & 2.236 & 1.361 & 2.189 \\
\hline 10 & 12 & 1.987 & 1.123 & 2.293 & 1.398 & 2.237 \\
\hline 20 & 15 & 1.994 & 1.127 & 2.342 & 1.482 & 2.301 \\
\hline 90 & 17 & 2.003 & 1.132 & 2.388 & 1.560 & 2.320 \\
\hline 36000 & 17 & 2.010 & 1.133 & 2.419 & 1.596 & 2.333 \\
\hline
\end{tabular}

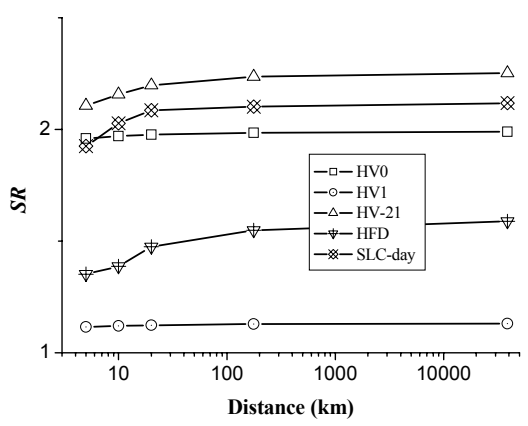

Fig. $9 S R$ vs. beacon distance for AO system sampling frequency of $2000 \mathrm{~Hz}$

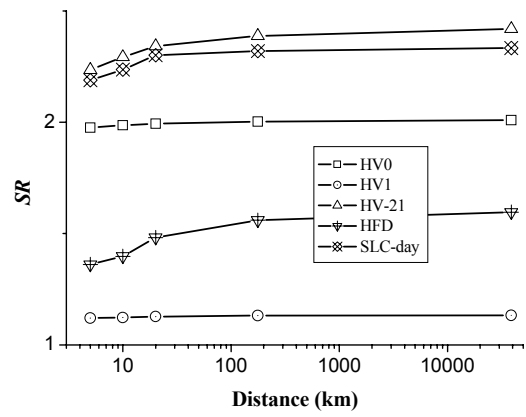

Fig. $10 S R$ vs. beacon distance for AO system sampling frequency of $4000 \mathrm{~Hz}$

(4) In the case of other wavelength

Choosing the light wavelength as $3.0 \mu \mathrm{m}, S R$ results are summarized in Table 10. Its corresponding figure is Fig. 11. It can be seen that in comparison to wavelength of $1.5 \mu \mathrm{m}$ (see Table 3 and Fig. 4), $r_{0-I R}$ becomes larger, i.e. the atmospheric turbulence strength becomes weaker and the phase compensation effectiveness $S R$ becomes apparently smaller. Further, it can be seen from Table 10 and Fig. 11 that the cone effect becomes less obvious as well.

(5) Further indication of effect of $C_{n}^{2}$ distribution vs. altitude

In order to understand influence of $C_{n}^{2}$ distribution vs. altitude on the cone effect better, two imaginary $C_{n}^{2}$ distributions are produced for models HV0 and SLC-day. In them it is assumed that $C_{n}^{2}$ is a constant at any altitude along the light path, i.e. $C_{n}^{2}$ distribution vs. altitude is even. At the same time, $r_{0}$ of the whole light path is maintained to be same as those in their original models. For the zenith angle of 0 degree and a wavelength of $1.5 \mu \mathrm{m}$, results for these two imaginary (even) distributions and their normal ones are summarized in Table 11. Its corresponding figure is Fig. 12. 
It can be seen from Table 11 and Fig. 12 that the cone effect becomes much larger for the even $C_{n}^{2}$ distributions. This is consistent with our explanation to the cone effect described above.

Table $10 S R$ vs. distance of the beacon in the case of a wavelength of 3.0 $\mathrm{\mu m}$, other conditions are same as Table 3

\begin{tabular}{|c|c|c|c|c|c|c|}
\hline $\begin{array}{c}\text { Distance } \\
(\mathrm{km})\end{array}$ & $\begin{array}{c}\text { Number } \\
\text { of PS }\end{array}$ & HV0 & HV1 & HV-21 & HFD & SLC-day \\
\hline \multicolumn{2}{|c|}{$R_{0-I R}(\mathrm{~cm})$} & 54.01 & 153.1 & 42.60 & 44.04 & 44.37 \\
\hline 5 & 9 & 1.195 & 1.022 & 1.293 & 1.141 & 1.313 \\
\hline 10 & 12 & 1.196 & 1.023 & 1.296 & 1.150 & 1.329 \\
\hline 20 & 15 & 1.197 & 1.024 & 1.299 & 1.157 & 1.338 \\
\hline 90 & 17 & 1.197 & 1.024 & 1.300 & 1.162 & 1.339 \\
\hline 36000 & 17 & 1.198 & 1.023 & 1.300 & 1.164 & 1.339 \\
\hline
\end{tabular}

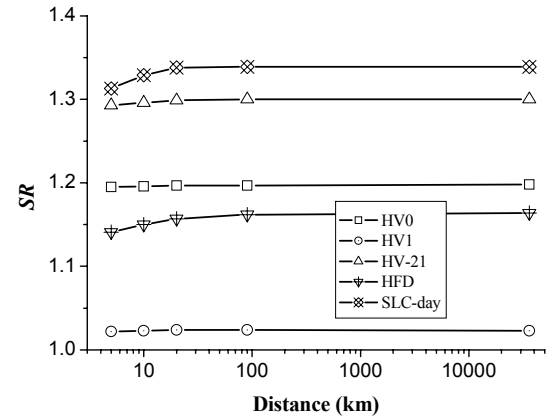

Fig. $11 S R$ vs. beacon distance for a wavelength of $3.0 \mu \mathrm{m}$

Table $11 S R$ vs. distance of the beacon under the same conditions as Table 2, showing influence of $C_{n}^{2}$ distribution vs. altitude

Wherein "Nor." denotes the normal $C_{n}^{2}$ distribution, and "Even" denotes an even (an imaginary) $C_{n}^{2}$ distribution

\begin{tabular}{|c|c|c|c|c|c|}
\hline \multirow{2}{*}{$\begin{array}{c}\text { Altitude } \\
(\mathrm{km})\end{array}$} & \multirow{2}{*}{$\begin{array}{c}\text { Number } \\
\text { of PS }\end{array}$} & \multicolumn{2}{|c|}{ HV0 } & \multicolumn{2}{c|}{ SLC-day } \\
\cline { 3 - 6 } & Nor. & Even & Nor. & Even \\
\hline 5 & 9 & 1.934 & 1.061 & 2.308 & 1.215 \\
\hline 10 & 12 & 1.943 & 1.126 & 2.423 & 1.307 \\
\hline 20 & 15 & 1.947 & 1.194 & 2.464 & 1.414 \\
\hline 90 & 17 & 1.949 & 1.431 & 2.485 & 1.768 \\
\hline 36000 & 17 & 1.949 & 1.437 & 2.502 & 2.102 \\
\hline
\end{tabular}

Fig. $12 S R$ vs. beacon altitude under the same conditions as Table 2, showing influence of $C_{n}^{2}$ distribution

\section{CONCLUSION}

By using an exemplary AO system, including the dynamic control process in a numerical simulation of the AO system, and proposing $S R$ as the evaluation parameter, the cone effect in astronomy with an AO system is investigated by means of a pure numerical simulation in this paper for the first time within our knowledge. Some conclusions can be deduced from this study: (1) It is surprisingly found that the effect of altitudes of the beacon (LGS or the astronomical target itself) on the $\mathrm{AO}$ phase compensation effectiveness by using the commonly-available vertical profiles of the refractive index structure constant $C_{n}^{2}$ and the lateral wind speed in the atmosphere is relatively weak, and the cone effect for some profiles is even negligible. (2) It is found that the cone effect does not have obvious relationship with the turbulence strength, however, it depends on the vertical distribution profile of $C_{n}^{2}$ apparently. On the other hand, the cone effect depends on the vertical distribution of the lateral wind speed as well. (3) The cone effect becomes more obvious as the zenith angle increases. (4) In comparison to a longer wavelength, the cone effect becomes larger in the case of a shorter wavelength. (5) In all cases concerned in this paper, an AO system by using the sodium guide star has almost same phase compensation effectiveness as that by using the astronomical target itself as a beacon. Although this 
study is carried out by utilizing an exemplary AO system and by using some concrete conditions, we believe that the conclusions deduced from it are commonly applicable.

\section{REFERENCE}

1. M. C. Roggemann and B. M. Welsh, Imaging Through Turbulence, CRC Press, Boca Raton, Florida, 1996.

2. R. K. Tyson, ed., Adaptive Optics Engineering Handbook, Dekker, New York, 2000.

3. R. Foy and A. Labeyrie, "Feasibility of adaptive optics telescope with laser probe," Astron. Astrophys. 152, pp. L29-L31, 1985.

4. N. Ageorges and J. C. Dainty, eds., Laser Guide Star Adaptive Optics for Astronomy, Kluwer Academic, Dordrecht, The Netherlands, 2000.

5. F. Roddier, ed., Adaptive Optics in Astronomy, Cambridge University press, 1999.

6. Feng Shen, "Technologies of laser guide star and low level light wavefront sensing for adaptive optics," Doctoral Thesis, Institute of Optics and Electronics, Chinese Academy of Sciences, 2002. (in Chinese)

7. E. Viard, M. Le Louarn and N. Hubin, "Adaptive optics with four laser guide stars: correction of the cone effect in large telescope," Appl. Opt. 41 (1), pp. 11-20, 2002.

8. D. F. Buscher, G. D. Love and R. M. Myers, "Laser beacon wave-front sensing without focal anisoplanatism," Opt. Lett. 27 (3), pp. 149-151, 2002

9. Hai-Xing Yan, Shu-Shan Li, De-Liang Zhang, and She Chen, "Numerical simulation of an adaptive optics system with laser propagation in the atmosphere," Appl. Opt. 39 (18), pp.3023-3031, 2000.

10. Hai-Xing Yan, She Chen, De-Liang Zhang, and Shu-Shan Li, "Numerical simulation of an adaptive optics system by means of modal wavefront reconstruction," Acta Optica Sinica 18 (1), pp. 103-108, 1998. (in Chinese)

11. Hai-Xing Yan, She Chen, and Shu-Shan Li, "Numerical simulation investigations of the effects of noise and detection error in an adaptive optics system," Proc. SPIE Vol. 4494, pp.144-155, 2002.

12. Hai-Xing Yan, Shu-Shan Li, and She Chen, "Numerical simulation investigations of the dynamic control process and frequency response characteristics in an adaptive optics system," Proc. SPIE Vol. 4494, pp.156-166, 2002.

13. Hai-Xing Yan, Shu-Shan Li, and She Chen, "Effects of reconstruction and controller coefficients on performances of an adaptive optics system investigated by a numerical simulation,” Proc. SPIE Vol. 4926, pp. 81-91, 2002.

14. She Chen, Hai-Xing Yan, and Shu-Shan Li, "Effects of noise and detection error in a dynamic adaptive optics system,” Proc. SPIE Vol. 4926, pp. 107-118, 2002.

15. Hai-Xing Yan, Shu-Shan Li, and She Chen, "preliminary numerical simulation investigation of the cone effect in an adaptive optics system using a laser guide star,” Proc. SPIE Vol. 5639, pp. 53-64, 2005.

16. Xinyang Li, Internal Report, Institute of Optics and Electronics, Chinese Academy of Sciences, Chengdu, 2002. (in Chinese) 